\title{
Novel Semi-open Technique for Repair of Acute Rupture of Achilles Tendon
}

\author{
${ }^{1}$ Sanjay Agarwala, ${ }^{2}$ Ganesh Mohrir, ${ }^{3}$ Anshul Shyam Sobti
}

\begin{abstract}
Background: The most common complication of open repair of Achilles tendon is skin necrosis and wound problems, especially in the distal part, where the blood supply to the skin is precarious. Minimally invasive and percutaneous techniques have become popular as they overcome this problem but these techniques put the sural nerve at risk and have high incidence of rerupture. We have devised a semi-open technique, which incorporates the advantages of both techniques and at the same time avoids their complications. The aim of this study was to evaluate the outcome of this technique.
\end{abstract}

Materials and methods: Twenty-two patients with acute Achilles tendon rupture were operated with this new technique. The operative technique included incision up to the tendon gap, no distal extension and a percutaneous passage of nonabsorbable suture through the distal stump with approximation and plantaris augmentation. The results were compared on the basis of functional results (Leppilahti score), cosmetic results, patient satisfaction and complications encountered.

Results: Fifteen patients had excellent (68.\%) and seven had good $(32 \%)$ results according to the Leppilahti score. Our average scar length was $6.2 \mathrm{~cm}$ and all patients were fully satisfied with their results and rehabilitation. There was one case of superficial infection and no cases of rerupture or sural nerve injury.

Conclusion: This new semi-open technique serves as a perfect combination of open and percutaneous surgeries, providing excellent functional and cosmetic results and minimizing the incidence of complications at the same time.

Keywords: Tendoachilles, Rupture, Repair, Percutaneous, Semi-open.

How to cite this article: Agarwala S, Mohrir G, Sobti AS. Novel Semi-open Technique for Repair of Acute Rupture of Achilles Tendon. J Foot Ankle Surg (Asia-Pacific) 2015;2(1):22-26.

Source of support: Nil

Conflict of interest: None

\section{INTRODUCTION}

Achilles tendon rupture is a common injury of the foot and ankle, with an increasing incidence in recent years. ${ }^{1,2}$

\footnotetext{
${ }^{1}$ Director (Professional Services) and Consultant

${ }^{2}$ Junior Consultant, ${ }^{3}$ Associate Consultant

${ }^{1-3}$ Department of Orthopedics, PD Hinduja National Hospital and Medical Research Centre, Mumbai, Maharashtra, India
}

Corresponding Author: Sanjay Agarwala, Director (Professional Services) and Consultant, Department of Orthopedics, PD Hinduja National Hospital and Medical Research Centre, Mumbai, Maharashtra, India, Phone: 9869446644, e-mail: drsa2011@gmail.com
There still exists a controversy regarding the ideal treatment method. ${ }^{3,4}$ Available options can be classified into following categories: conservative treatment, open surgery, and minimally invasive repair. ${ }^{4,5}$

Systematic review of the literature, recommends surgical treatment as the optimal strategy with particular attention paid to rerupture and complications. ${ }^{6}$ High risk of infection, skin necrosis and scar problems of open repair ${ }^{5,7}$ led researchers to devise minimally invasive and percutaneous methods of repair. At present, with the percutaneous suture techniques the functional recovery is almost identical to that of open surgery but with a significantly lower incidence of postoperative complications. ${ }^{4}$ However, these methods persist to have complications like rerupture ${ }^{8-10}$ and sural nerve damage. .,11 $^{-1}$

Instead of using the typical percutaneous suture technique, we have devised a semi invasive technique combining the advantages and minimizing the complications of both techniques. We use a open technique in the proximal part of the repair while a percutaneous repair in the distal part, which is notorious for its poor blood supply and wound healing problems. ${ }^{12}$ In this study, we have analyzed the results of this technique and compared it with world literature of both open and minimally invasive techniques.

\section{MATERIALS AND METHODS}

\section{General Information}

Twenty-nine patients with acute rupture of Achilles tendon of less than 7 days duration presented to our clinic between August 2005 and May 2012. Adult patients less than 60 years of age, with no medical morbidity and a previously active lifestyle were included in our study. Pathological ruptures, incomplete tears and open injuries were excluded from the study. Seven patients did not fit the inclusion criteria. We evaluated 22 patients based on our inclusion criteria. Patients were diagnosed based on history, clinical examination and MRI confirmation. Demographic data are as shown in Table 1.

\section{Operative Technique}

All patients underwent surgery under general anesthesia with tourniquet control in prone position. A paramedial incision to tendo Achilles tendon was taken. The 
Table 1: Demographic data

\begin{tabular}{ll}
\hline $\begin{array}{l}\text { Age at operation (mean; range) (years) } \\
\text { Male: female (mean; range) (no. of }\end{array}$ & $45.6(26-58)$ \\
patients) & $20: 2$ \\
$\begin{array}{l}\text { Delay before repair in hours after injury } \\
\text { (mean; range) }\end{array}$ & 41.4 hours (18-60) \\
$\begin{array}{l}\text { Follow-up period in months } \\
\text { (mean; range) }\end{array}$ & 53 months (33-78) \\
Preinjury activity level & \\
- Competitive athlete & 04 \\
- Recreational athlete & 07 \\
- Non-athlete & 11 \\
Injured side (right:left) (no. of patients) & $14: 8$ \\
Cause of injury & 11 \\
- Sports & 11 \\
- Minor trauma &
\end{tabular}

Minor trauma: Missing a step, fall and give away on Indian style squatting, etc.

incision was not extended beyond the proximal aspect of the palpable gap and distally up to proximal extent of distal stump (Fig. 1). Paratenon was incised and the ruptured ends were debrided. The plantaris tendon was isolated and cut proximally with the distal insertion left intact. Nonabsorbable sutures were passed with modified Kessler's stitch through the proximal stump and
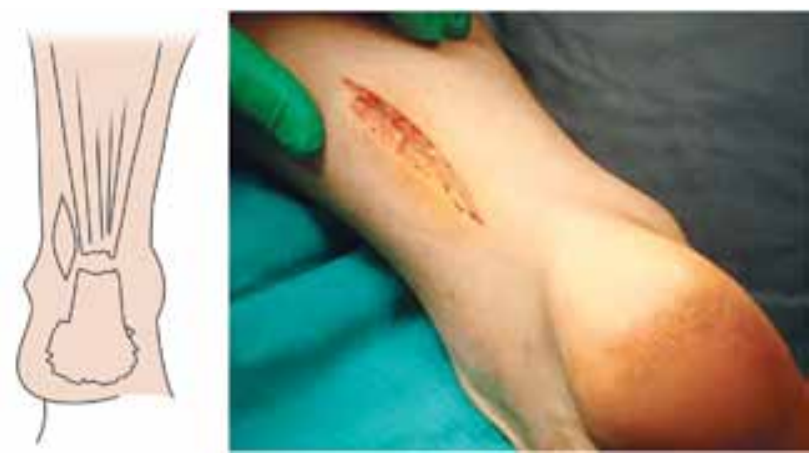

Fig. 1: Medial skin incision (strictly above the rupture site)

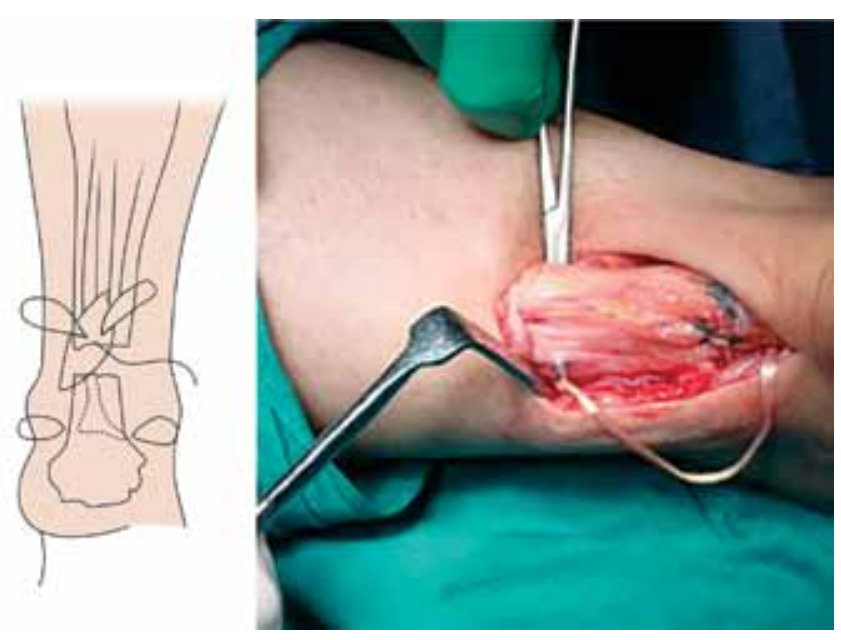

Fig. 3: Suture configuration prior to approximation percutaneously in the distal stump firstly directed medially inside out from the stump to skin, and then outside in through the same punctured site (Fig. 2). The suture was passed laterally through the stump and the same procedure repeated to complete the suture configuration (Fig. 3). Suture was tightened with knee flexed and ankle plantar flexed. Tension was applied to plantaris tendon which was weaved across the repaired junction, helping augment the repair (Fig. 4). The paratenon was sutured with 5-0 ethibond ${ }^{\mathrm{TM}}$ continuous sutures. Skin was approximated with interrupted sutures.

\section{Postoperative Care}

Postoperatively-the limb was placed in an below knee plaster in 200 of equinus for 14 days. After 14 days, below knee plaster with ankle in neutral position for 2 weeks. Following this plaster was removed and ankle was mobilized, patients were allowed initially partial weight bearing to full weight bearing with wedges in the foot wear. Patients were allowed to return to full activities at 8 to 10 weeks. Patients were also referred for physiotherapy for range of movement exercises and later muscle strengthening exercises.

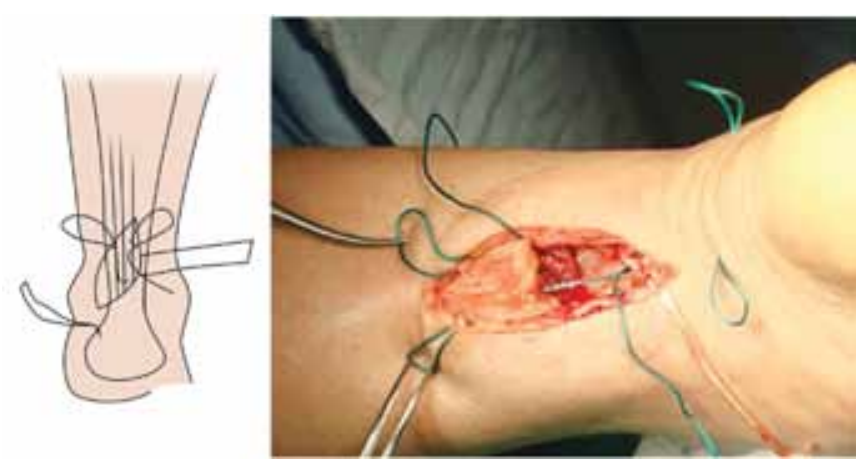

Fig. 2: Percutaneous passage of distal suture (inside out technique)

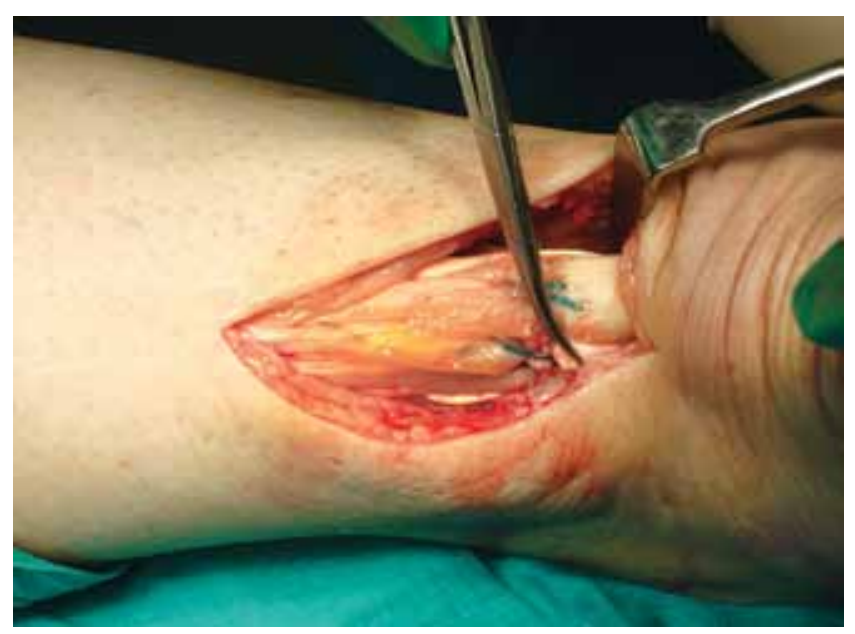

Fig. 4: Plantaris augmentation with complete suture configuration 


\section{Follow-up Assessment and Outcome Evaluation}

The patients were followed up at regular intervals at 6 , 12, 24 weeks and thereafter yearly. Functional outcome was assessed at follow-up visits with use of the clinical scoring method described by Leppilahti et al. ${ }^{13}$ The scoring included subjective factors, such as pain, stiffness, muscle weakness, footwear restrictions, and subjective outcome as well as objective factors, such as the active range of ankle motion and isokinetic calf muscle strength. The maximum number of points achievable was 100 .

Table 2: Results

\begin{tabular}{ll}
\hline - Pain & \\
- None & 16 \\
- Mild, no limitation of recreational activities & 06 \\
- Moderate, limitation on daily activities but not on & 0 \\
& recreational activities \\
- Severe, limitation of daily and recreational activity & 0 \\
- Early failure & 0
\end{tabular}

- Stiffness

- None

- Mild, no limitation of recreational activities

- Moderate, limitation on daily activities but not on recreational activities

- Severe, limitation of daily and recreational activity

- Early failure

- Calf muscle weakness (subjective)

- None

- Mild, no limitation of recreational activities

- Moderate, limitation on daily activities but not on recreational activities

- Severe, limitation of daily and recreational activity 0

- Early failure

- Footwear restrictions

- None

- Mild, most shoes tolerated

- Moderate modified shoes tolerated

- Early failure

- Active range of movement difference between ankles (no. of patients)

- Normal $<5^{\circ}$

- Mild $6^{\circ}$ to $10^{\circ}$

- Moderate $11^{\circ}$ to $15^{\circ}$

- Severe $>16^{\circ}$

- Early failure

- Subjective results

- Very satisfied

- Satisfied, with minor reservations

- Satisfied, with major reservations

- Dissatisfied

- Early failure

- Leppilahti score

- Excellent

- Good

- Fair

- Poor

- Early failure
The result was classified as excellent ( $\geq 90$ points), good (75-89 points), fair (60-74 points), or poor ( $<60$ points). Cosmetic results were assessed on the basis of scar length. Patient satisfaction was assessed by the answers given to a non-validated subjective symptoms questionnaire. A subjective result 'satisfied but with major reservations' indicated that the patient was satisfied in the activities of daily living but that the recreational activity level was reduced. All complications encountered were duly noted with special attention to wound healing, skin necrosis, sural nerve injury and reruptures.

\section{RESULTS}

\section{General Information}

The average duration of surgery was 53.8 minutes (44-70 minutes). The average tourniquet time was 44.4 minutes (36-62 minutes). The average length of the incision was $6.2 \mathrm{~cm}(5-8.1 \mathrm{~cm})$.

\section{Functional Outcome}

The Leppilahti score at the time of 2 years follow-up was the primary outcome measure. In the series, this score was classified as excellent for 15 patients $(68.18 \%)$ and good for seven (31.81\%). Table 2 shows the assessment outcome at 2 years in terms of the subjective result, pain, stiffness, subjective calf muscle weakness, footwear restrictions, or the range of ankle motion. These parameters are used in the Leppilahti score calculation. The average time to return to daily activities was 10.2 weeks (8-12.5 weeks).

\section{Complications}

One patient had superficial infection. The patient was a 36-year-old male who had clear drainage from the incision site at 2 weeks. Cultures of specimens from the wound were negative. The patient received 7 days of oral antibiotics and the wound healed without further evidence of infection. The patient was able to walk normally eventually. There were no cases of skin necrosis, sural nerve affection or reruptures.

\section{DISCUSSION}

It is well established among clinicians that conservative treatment of closed Achilles tendon rupture is associated with high morbidity. Although open surgery is the preferred option, the outcomes vary greatly. Surgical management of Achilles tendon rupture has been practiced since the 10th century in Arabia. ${ }^{5}$ In 1888, Frenchman Gustave Polaillon ${ }^{14}$ first described the technique of open repair and the technique has been progressively 
advanced through the ages. Even with the advancements, open repair has been plagued by several complications of wound healing and skin necrosis. ${ }^{5,7}$ One of the reasons cited for the high rate of skin necrosis is the precarious blood supply to the skin around the distal attachment of the tendon. ${ }^{12}$ To over come this issue Ma and Griffith in 1960 first came up with the percutaneous repair of the tendon. ${ }^{15,16}$ Their technique being percutaneous avoided any vascular compromise to the skin and had significantly decreased wound complications. However with more and more surgeons adapting this technique, there was a high incidence of sural nerve injury ${ }^{8,11}$ and reruptures. 8,10

Recent meta-analyses reported rerupture rates for surgically treated patients varying from 3.1 to $5.0 \%$ vs 8.8 to $13 \%$ for nonsurgically treated patients. ${ }^{6,17}$ Some authors reported favorably toward the percutaneous approaches in terms of complication rates, but a higher rate of sural nerve injury was noted. ${ }^{6,18}$ Klein et al $^{15}$ showed an incidence of $13 \%$ of sural nerve injuries. In our study there was no sural nerve injury.

A meta-analysis of randomized controlled trials comparing open surgery to percutaneous surgery, found a significantly higher rate of deep infections with open surgery (19.6 vs 0\% with percutaneous surgery). ${ }^{19}$ The difference in the rate of rerupture $(4.3 \%$ with open surgery vs $2.1 \%$ with percutaneous surgery) was not statistically significant.

Delponte ${ }^{20}$ showed rerupture rates of $10 \%$ for percutaneous repair which were similar to nonoperative repair. This can be attributed to the fact that lack of visualization and nonanatomic end to end apposition in percutaneous technique results in healing by deposition of fibrous tissue. ${ }^{21}$ This fibrous tissue has been shown to be collagen type 3 and not the normal type 2 collagen and structurally weaker, and hence more prone to re rupture. ${ }^{22}$

Our series showed excellent results with our functional results being at par if not better than others, including both open and minimally invasive techniques. ${ }^{5,7,9-11}$ Along with this we had minimal wound complications (4.5\%), compared to an incidence of $14 \%$ described in literature. ${ }^{23}$ We attribute this to the fact that we restrict our incision proximal to the rupture point thus avoiding any incision and dissection on the distal skin with the precarious blood supply. At the same time, the proximal incision gives us enough space to visualize and debride the rupture gap ensuring an anatomical end to end repair. Our series had no reruptures at the end of 2 years follow-up which is again better than most of the other series. ${ }^{15,19,20}$ The average length of incision was $6.2 \mathrm{~cm}$ and on the medial aspect. None of our patients had any cosmetic complains about the scar. At the same time, our incision is long enough to prevent any inadvertent injury to the sural nerve.

Our study has limitations of being retrospective and that it lacks a control group. However, it involves a detailed analysis and comparisons with both open and minimally invasive techniques. In conclusion we believe that our original minimally invasive tendon skin suture technique combines the advantages of both the percutaneous suture and open direct suture techniques. It complies with the anatomy and physiology of the Achilles tendon, and thus provides an optimal environment for healing.

\section{REFERENCES}

1. Fu C, Qu W. Acute Achilles tendon rupture: mini-incision repair with double-tsuge loop suture vs open repair with modified kessler suture. The Surgeon. Elsevier Ltd; 2015 Jan;31:1-6.

2. Jarvinen TAH, Kannus P, Maffulli N, Khan KM. Achilles tendon disorders: etiology and epidemiology. Foot Ankle Clin 2005 Jun;10(2):255-266.

3. Lo IK, Kirkley A, Nonweiler B, Kumbhare DA. Operative versus nonoperative treatment of acute Achilles tendon ruptures: a quantitative review. Clin J Sport Med 1997 Jul; 7(3):207-211.

4. Ding W-G, Li H, Zhu Y-P, Liu Z-W. Comparison between tenocutaneous suture and kessler suture techniques in treating acute closed Achilles tendon rupture. Foot and Ankle Surgery 2014 Jun;20(2):105-108.

5. Maffulli N. Current concepts review-rupture of the Achilles tendon. J Bone and Joint Surg 1999.

6. Bevoni R, Angelini A, D'Apote G, Berti L, Fusaro I, Ellis S, et al. Long-term results of acute Achilles repair with triplebundle technique and early rehabilitation protocol. Injury Elsevier Ltd; 2014 Aug 1;45(8):1268-1274.

7. Arner O, Lindholm A. Subcutaneous rupture of the Achilles tendon; a study of 92 cases. Acta Chir Scand Suppl 1958.

8. Carmont MR, Rossi R, Scheffler S, Mei-Dan O, Beaufils P. Percutaneous and mini invasive achilles tendon repair. Sports Med Arthrosc Rehabil Ther Technol 2011 Nov;3:28.

9. Pina A, Lozano JA, Torro V, Escriba I. Percutaneous suture of achilles tendon ruptures. Foot Ankle 1992.

10. Nistor L. Surgical and nonsurgical treatment of Achilles tendon rupture. J Bone Joint Surg A 1981.

11. Hockenbury RT, Johns JC. A biomechanical in vitro comparison of open versus percutaneous repair of tendon Achilles. Foot Ankle 1990 Oct.;11(2):67-72.

12. Haertsch PA. The blood supply to the skin of the leg: a postmortem investigation. Br J Plast Surg 1981 Oct;34(4):470-477.

13. Leppilahti J, Forsman K, Puranen J, Orava S. Outcome and prognostic factors of Achilles rupture repair using a new scoring method. Clin Orthop Relat Res 1998 Jan;(346): 152-161.

14. Carlstedt CA. Mechanical and chemical factors in tendon healing. Effects of indomethacin and surgery in the rabbit. Acta Orthop Scand Suppl 1987;224:1-75.

15. Klein W, Lang DM, Saleh M. The use of the Ma-Griffith technique for percutaneous repair of fresh ruptured tendo Achillis. Chir Organi Mov 1991 Jul;76(3):223-238. 
16. Lacoste S, Féron JM, Cherrier B. Percutaneous tenolig ${ }^{\circledR}$ repair under intraoperative ultrasonography guidance in acute Achilles tendon rupture. Orthopaedics and traumatology: surgery and research. Elsevier Masson SAS 2014 Dec 1;100(8): 925-930.

17. Khan RJ, Carey Smith RL. Surgical interventions for treating acute Achilles tendon ruptures. Cochrane Database Syst Rev 2010;(9):CD003674.

18. Gigante A, Moschini A, Verdenelli A, Del Torto M, Ulisse S, de Palma L. Open versus percutaneous repair in the treatment of acute Achilles tendon rupture: a randomized pros -pective study. Knee Surg Sports Traumatol Arthrosc 2008 Feb;16(2):204-209.

19. Khan RJK, Fick D, Keogh A, Crawford J, Brammar T, Parker M. Treatment of acute Achilles tendon ruptures. A meta-analysis of randomized, controlled trials. J Bone Joint Surg Am 2005 Oct;87(10):2202-2210.

20. Delponte P, Potier L, de Poulpiquet P, Buisson P. Treatment of subcutaneous ruptures of the Achilles tendon by percutaneous tenorraphy. Rev Chir Orthop Reparatrice Appar Mot 1992;78(6):404-407.

21. Suchak AA, Spooner C, Reid DC, Jomha NM. Postoperative rehabilitation protocols for Achilles tendon ruptures: a metaanalysis. Clin Orthop Relat Res 2006 Apr;445:216-221.

22. Sundaram N, Sakthivel K, Kumar S. Traumatic rupture of the Achilles tendon in people using Indian styled toilets: an observation. Ind J Orthop 2001.

23. Wong J, Barrass V, Maffulli N. Quantitative review of operative and nonoperative management of Achilles tendon ruptures. Am J Sports Med 2002 Jul;30(4):565-575. 